\title{
Modulation of gut mucosal biofilms
}

\author{
Brigitta Kleessen $^{1 *}$ and Michael Blaut ${ }^{2}$ \\ ${ }^{1}$ Veterinary Faculty, Institute of Bacteriology and Mycology, University of Leipzig, An den Tierkliniken 29, D-04103 Leipzig, Germany \\ ${ }^{2}$ German Institute of Human Nutrition (DIFE) Potsdam-Rehbrücke, D-14558 Nuthetal, Germany
}

\begin{abstract}
Non-digestible inulin-type fructans, such as oligofructose and high-molecular-weight inulin, have been shown to have the ability to alter the intestinal microbiota composition in such a way that members of the microbial community, generally considered as health-promoting, are stimulated. Bifidobacteria and lactobacilli are the most frequently targeted organisms. Less information exists on effects of inulin-type fructans on the composition, metabolism and healthrelated significance of bacteria at or near the mucosa surface or in the mucus layer forming mucosa-associated biofilms. Using rats inoculated with a human faecal flora as an experimental model we have found that inulin-type fructans in the diet modulated the gut microbiota by stimulation of mucosa-associated bifidobacteria as well as by partial reduction of pathogenic Salmonella enterica subsp. enterica serovar Typhimurium and thereby benefit health. In addition to changes in mucosal biofilms, inulin-type fructans also induced changes in the colonic mucosa stimulating proliferation in the crypts, increasing the release of mucins, and altering the profile of mucin components in the goblet cells and epithelial mucus layer. These results indicate that inulin-type fructans may stabilise the gut mucosal barrier. Dietary supplementation with these prebiotics could offer a new approach to supporting the barrier function of the mucosa.
\end{abstract}

Bifidobacteria: Intestinal biofilms: Inulin-type fructans: Mucosa: Gnotobiotic rats

The human gut ecosystem consists of a variety of different habitats and metabolic niches that are colonised by the so-called microbiota that contain more than $10^{11}$ micro-organisms per gram wet weight of contents, predominantly anaerobes (Macfarlane et al. 1997). The intestinal microbiota has both beneficial and pathogenic potential (Fuller \& Gibson, 1997). The microbial community can provide protection against pathogenic bacteria, stimulates cell-mediated and humoral immune responses, and indirectly supports digestive processes by microbial fermentation (Berg, 1996; Cummings \& Macfarlane, 1997). It also includes potential pathogenic organisms such as certain species of Clostridium, Escherichia, Salmonella, Shigella and Pseudomonas, as well as yeasts such as Candida albicans (Salminen et al. 1995). Evidence exists that the best protection against mucosal attachment and invasion by such pathogens is by keeping intestinal microbiota in a state that affords colonisation resistance against pathogens by modulation of the microbiota and by inducing luminal or systemic effects which are beneficial to the host's health. This may be achieved by the consumption of non-digestible food ingredients such as inulin-type fructans, known as prebiotics, which favour the growth and activity of certain colonic bacteria, such as bifidobacteria and lactobacilli, generally regarded as beneficial to the host (Gibson et al. 1995; Kleessen et al. 1997). Effects of inulin-type fructans on the luminal flora in the gut of human subjects or rats are known (Gibson et al. 1995; Campbell et al. 1997; Djouzi \& Andrieux, 1997; Kleessen et al. 1997; Bouhnik et al. 1999; Kruse et al. 1999; Le Blay et al. 1999; Kleessen et al. 2001). However, less information exists on how inulin-type fructans modulate the interaction between gut morphology, mucosa-associated microbiota and health of the host. Therefore, we studied the effects of inulin-type fructans on bacteria being present at or near the mucosa surface or in the mucus layer forming mucosa-associated biofilms.

\section{Spatial organisation of microbial communities in the gastrointestinal tract}

Biofilms are complex microbial communities attached to surfaces. Our understanding of the complex structure and function of bacterial biofilms in the gastrointestinal tract and on their metabolic significance for the host is still far from complete and requires novel approaches in research. It is important to get more information on colonisation factors such as adhesive properties, surface components and utilization of substrate and growth rate of intestinal bacteria. In the gastrointestinal tract bacteria may be free-living or attached to mucus, mucosa surface, food particles or digestive residues (Macfarlane et al. 1999). The attached bacteria produce microcolonies, leading to the development of biofilms, which initially may be composed of only one bacterial species, but frequently develop into a complex community composed of different bacterial species. A schematic illustration of possible stages of biofilm development is shown in Fig. 1. Mucins, other secretory proteins or polymers mainly composed of polysaccharides cover mucosal surfaces in the intestine and are therefore potential adherence sites for intestinal bacteria (Freitas \& Cayuela, 2000). While these polymers may form a barrier against colonisation by some bacteria, other bacteria can use them as a means to adhere to the surface. Adherence leads 


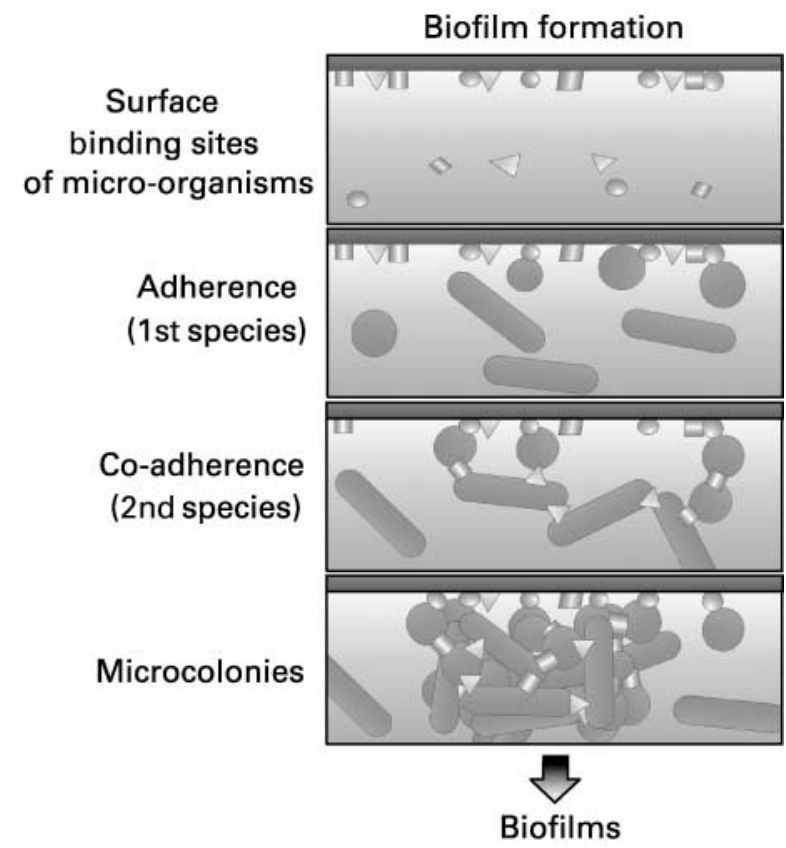

Fig. 1. Generalised scheme depicting the development of gut biofilms.

to the formation of an adhesive microbial layer of one species that subsequently may support colonisation of other micro-organisms through co-adherence, promoting the development of microcolonies and biofilms. Interactions among populations of such communities are complex and both the microbial-microbial and the microbial-host relationships are dependent on intra- and inter-species communication systems (Hooper \& Gordon, 2001).

\section{The gut mucosal barrier}

The interaction of bacteria with the mucus layer and/or with the intestinal mucosa is important because the first contact of bacteria with the intestinal tissue is with the mucus layer, which covers the underlying epithelium, consisting of enterocytes, columnar in shape, with an apical and a basolateral side and microvilli on the apical side (Fig. 2). The mucus gel is formed by high-molecularweight mucins secreted by the goblet cells and serves as a habitat for the resident microflora by providing a specific microenvironment (Forstner, 1978). The amount and composition of the mucus layer reflect the balance between mucus secretion, and its erosion and degradation by bacteria (Deplancke \& Gaskins, 2001). In addition, other glycoconjugates are also present; mainly glycoproteins and glycolipids synthesised by most of the epithelial cells producing the glycocalyx (Freitas \& Cayuela, 2000). Such mucins and glycoconjugates play a key role in the barrier function. This means that the intact intestinal mucosa covered with a biofilm of non-pathogenic bacteria represents a barrier to the unrestrained uptake of antigens and pro-inflammatory molecules, including bacteria and bacterial products (Fig. 2). When the normal microflora, the mucus layer or the epithelial cells are disturbed by pathogens, antigens and other toxic substances from the gut lumen, defects in the barrier system become evident (Neish, 2002). Such a compromised mucosal barrier may increase paracellular permeability of the mucosa. As a consequence, the probability of bacterial invasion of the intestinal mucosa is increased under these conditions, resulting in inflammatory and immunologic responses (Lu \& Walker, 2001).

Therefore, the best protection against mucosal attachment and invasion by pathogens or other harmful substances could be the maintenance of the normal microbiota adhering tenaciously

\section{Intact mucosal barrier}

\section{Disturbed mucosal barrier}
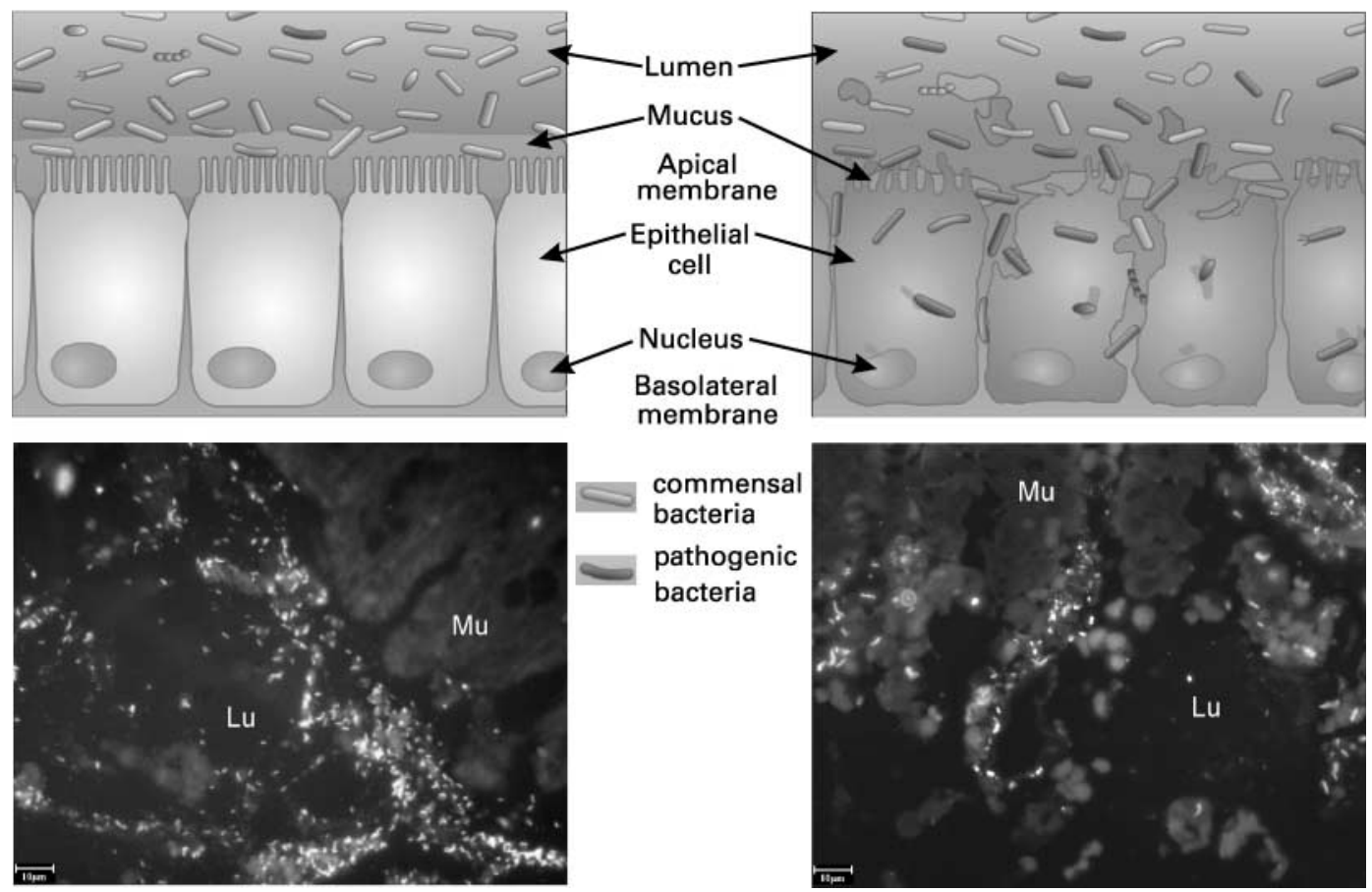

membrane

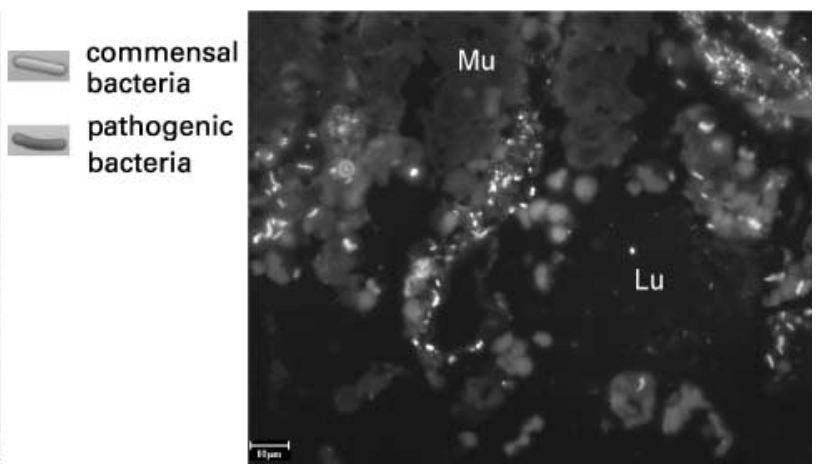

Fig. 2. Schematic illustration of an intact and a disturbed mucosal barrier (upper drawings). The lower micrographs show bacteria in human colonic tissue sections detected with a mixture of fluorescently labelled 16S rRNA-targeted oligonucleotide probes, specific for Bacteria mucosa of a healthy (left) and a colitic human subject (right). The oligonucleotides were $5^{\prime}$ labelled with the indocarbocyanine dye Cy3. Mu, mucosa; Lu, lumen. 
to mucus overlaying the mucosa. Moreover, consumption of inulin-type fructans could be one way to modulate mucosa-associated biofilms so that members of the biofilm community, generally considered as health-promoting, are stimulated.

\section{Methods for assessing the intestinal biofilms}

To be certain that the consumption of inulin-type fructans in the diet modulate intestinal microflora in a targeted way, the exact qualitative and quantitative monitoring of changes in the intestinal microbiota is an essential prerequisite. Therefore, methods for the characterisation of micro-organisms in situ and for the determination of their microbial activity within the natural habitat are preferred (Amann et al. 1995; Amann \& Kühl, 1998). Fluorescent in situ hybridisation (FISH) ith 16S/23S rRNA-targeted oligonucleotide probes in combination with epifluorescence and/or laser scanning microscopy has made it possible to study the composition of the intestinal microflora in a culture-independent way both in the intestinal content (luminal bacteria) and in the tissue sections (mucosa-associated bacteria; Franks et al. 1998; Kleessen et al. 2002). Moreover, this technique is required for the direct detection of the spatial organisation of microbial communities in intestinal biofilms (Tolker-Nielsen \& Molin, 2000).

In general, FISH represents a rapid, reliable and accurate method for studying the microbial populations of intestinal microbiota. Briefly, for whole-cell hybridisation, the bacteria have to be fixed and permeabilised with paraformaldehyde or ethanol to allow the oligonucleotide probes to reach their target sequence of the ribosomal RNA. When the sequence of the fluorescent probe used is complementary to a target sequence of the cells in the sample, hybrids are formed and the fluorescing cells can be viewed by epifluorescence microscopy (Amann et al. 1990).

\section{Inulin-type fructans modulate the gut mucosal biofilms in} human flora-associated rats

We studied the effects of inulin-type fructans in the diet on numbers of luminal and mucosa-associated bacteria (total bacteria, bifidobacteria, Bacteroides/Prevotella) in the distal jejunum and the distal colon of male 5-week-old germ-free rats (Wistar, inbred strain AVN-Ipcv; German Institute of Human Nutrition) inoculated with a fresh human faecal flora (HFA, human floraassociated; Kleessen et al. 2003). A $10 \mathrm{~d}$ after inoculation rats were allocated to one of the two dietary treatments for $28 \mathrm{~d}$ (groups of six rats). Dietary treatments were a commercial standard (ST) diet (Altromin fortified ${ }^{\circledR} 1310$; Altromin, Lage, Germany) or ST supplemented with a mixture of oligofructose (OF) and highmolecular-weight inulin (inulin HP). FISH with appropriate 16S rRNA-targeted probes was used to monitor changes in bacterial population groups both in intestinal contents and in tissue sections in response to feeding these diets (Kleessen et al. 2003).

One important finding of our study concerns the increase in colonic mucosal bifidobacteria in rats consuming the diet containing the mixture OF/inulin HP compared with ST. Such stimulation was not observed in the intestinal lumen of the HFA rats (Fig. 3). In contrast, only a slight trend towards lower counts of Bacteroides/Prevotella was observed on the colonic mucosa of HFA rats fed inulin-type fructans compared with those fed ST (Fig. 4). The numbers of total bacteria were unaffected by the diet both in the lumen and on the mucosa. In view of these findings, the higher number of mucosa-associated bifidobacteria of rats fed inulin-type fructans led as to speculate that these organisms might contribute to the protection of the intestinal mucosa. A logical next step would be to define the exact mechanisms underlying the claimed health-promoting effects of mucosal bifidobacteria.

\section{Inulin-type fructans stabilise the gut mucosal barrier in human flora-associated rats}

With a view towards the increased bifidobacterial numbers on the colonic mucus layer by inulin-type fructans, the question arose whether fructans also have an effect on mucosal morphometry. Therefore, changes in the mucosal architecture (height of villi, depth of crypts and number of goblet cells) were studied on stained tissue sections (haematoxylin and eosin) by light microscopy and image analysis (Kleessen et al. 2003). As expected, we found that villus height and crypt depth were significantly higher in HFA rats consuming the mixture OF/inulin HP than in rats consuming ST (Fig. 5). These modifications could have consequences on the intestinal physiology of the

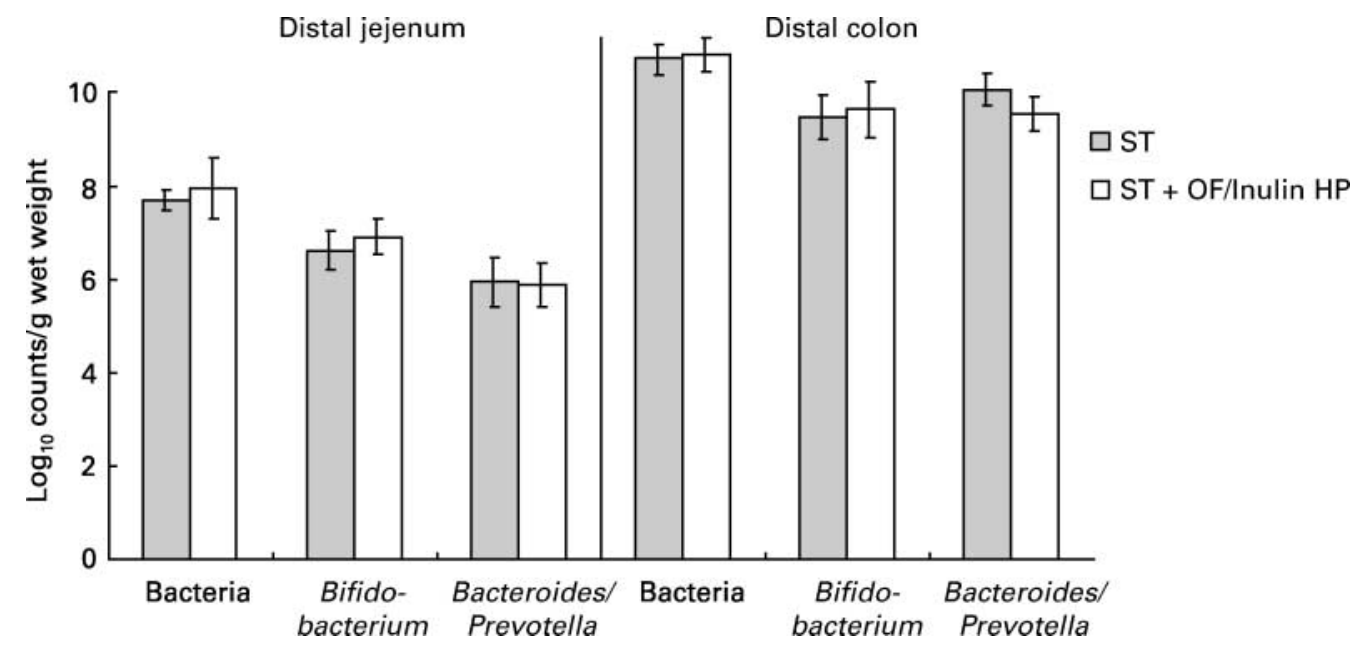

Fig. 3. Numbers of bacteria in the contents of the distal jejunum and the distal colon of human flora-associated rats fed a standard diet (ST) or a standard diet supplemented with inulin-type fructans (OF, oligofructose; Inulin HP, high-molecular-weight inulin). 


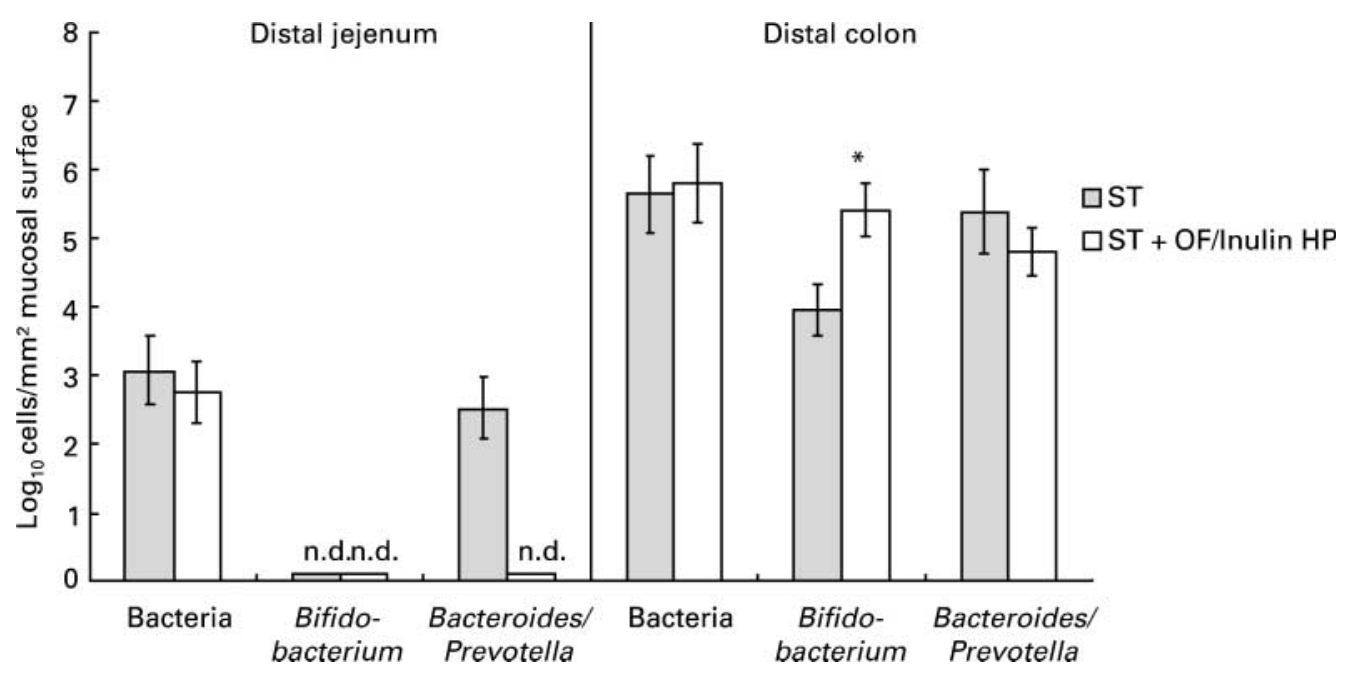

Fig. 4. Numbers of mucosa-associated bacteria in the distal jejunum and the distal colon of human flora-associated rats fed a standard diet (ST) or a standard diet supplemented with inulin-type fructans (OF, oligofructose; Inulin HP, high-molecular-weight inulin). Mean values were significantly different from those of the ST group: ${ }^{*} P<0.05$. n.d., not determined.

host. It is assumed that an increased villus height is paralleled by an increased digestive and absorptive function of the intestine due to increased expression of brush border enzymes and nutrient transport systems (Pluske et al. 1996). Moreover, as inulin-type fructans are not absorbed in the small intestine but fermented in the colon, the resulting bacterial fermentation products such as SCFA are the preferred energy substrates of the colonocytes and have a trophic effect on the mucosa (Sakata, 1987).

Besides the changes in the mucosal architecture we expected changes in the thickness of the mucus layer covering the mucosa and/or in numbers of goblet cells in response to feeding of inulin-type fructans. Studies on stained tissue sections of the above HFA rats have shown that the addition of the mixture OF/inulin HP to the diet resulted in a thicker colonic epithelial mucus layer compared with control rats $(62.2 \pm 9.9 v$. $43 \cdot 4 \pm 7 \cdot 1 \mu \mathrm{m})$. Compared with ST, inulin-type fructans also increased the number of goblet cells in the crypts of HFA rats (distal jejunum: $23 \pm 5$ v. $17 \pm 4$; distal colon: $32 \pm 4$ v. 24 \pm 6 ). To identify different mucin components in the goblet cells and the epithelial mucus layer, serial sections of the intestinal tissue were subjected to the following stainings: (i) the periodic acidSchiff reaction for unsubstituted $\alpha$-glycerol rich neutral mucins,

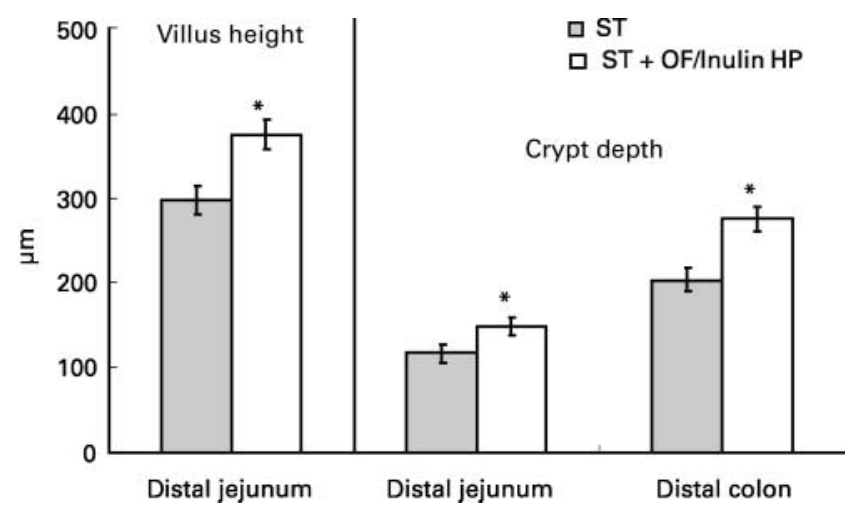

Fig. 5. Increase of villus height and crypt depth in the distal jejunum and distal colon of human flora-associated rats by inulin-type fructans in the diet (OF, oligofructose; Inulin HP, high-molecular-weight inulin). Mean values were significantly different from those of the ST group: ${ }^{*} P<0.05$. (ii) Alcian Blue $(\mathrm{AB}) \mathrm{pH} 2.5$ to identify acidic mucins and (iii) $\mathrm{AB} \mathrm{pH} 1.0$ to differentiate sulphated mucins. Mucins stained by $\mathrm{AB} 2 \cdot 5$, but not by $\mathrm{AB} 1 \cdot 0$, were classified as sialomucins (Romeis, 1989). Sulphomucins were the predominant type of acid mucins in the colonic mucosa of rats fed the mixture $\mathrm{OF} /$ inulin HP, while sialomucins dominated in the colon of rats fed ST (Kleessen et al. 2003). Fontaine et al. (1996) have reported similar results. Such differences in the amount of sulphomucins and sialomucins could have important effects on the susceptibility of epithelial mucins for degradation by bacterial enzymes (Rhodes, 1997) as it has been assumed that changes in the thickness or composition of the mucus layer covering the epithelium facilitates bacterial invasion into the inflamed mucosa (Pullan et al. 1994). Therefore, stabilisation of the gut mucosal barrier, either by the increased release of mucins and/or by the stimulation of sulphomucins with inulin-type fructans, could become

Table 1. Effect of inulin-type fructans on Salmonella numbers in intestinal contents and tissues of human flora-associated (HFA) rats after oral administration of Salmonella enterica subsp. enterica serovar Typhimurium*

\begin{tabular}{|c|c|c|}
\hline \multirow[b]{2}{*}{ Diet† } & \multicolumn{2}{|c|}{ Salmonella } \\
\hline & Standard diet & $\begin{array}{l}\text { Standard diet }+ \\
\text { OF/Inulin HP }\end{array}$ \\
\hline \multicolumn{3}{|l|}{ Ileum } \\
\hline Content & $3.7(0.7) \ddagger$ & $<1.0$ \\
\hline Mucus & $3.5(0.5)$ & $3.2(0.8)$ \\
\hline Tissue & $4.0(0.4)$ & $3.2(0.7)$ \\
\hline \multicolumn{3}{|l|}{ Cecum } \\
\hline Content & $4.8(0.9)$ & $<1.0$ \\
\hline \multicolumn{3}{|l|}{ Colon } \\
\hline Content & $4.9(0.6)$ & $2 \cdot 3$ (only one rat) \\
\hline Mesenteric lymph nodes & $5.8(0.6)$ & $5.4(0.3)$ \\
\hline Peyer's patches & $5.7(0.4)$ & $4.4(0.6) \S$ \\
\hline \multicolumn{3}{|c|}{$\begin{array}{l}\text { *HFA rats were killed } 15 \mathrm{~d} \text { after oral infection with a single dose of } S \text {. Typhimurium } \\
\left(4 \times 10^{8} \text { viable cells). }\right.\end{array}$} \\
\hline \multicolumn{3}{|c|}{$\begin{array}{l}\text { †HFA rats were fed either a standard diet or a standard diet supplemented with } 50 \mathrm{~g} \text { fruc- } \\
\text { tans }\left(25 \mathrm{~g} \text { oligofructose (OF; Raftilose }{ }^{\circledast} \text { P95 Raffinerie Tirlemontoise, Tienen, Belgium) }\right. \\
+25 \mathrm{~g} \text { high-molecular-weight inulin (inulin HP; Raftiline }{ }^{\circledR} \mathrm{HP} \text {, Raffinerie Tirlemon- } \\
\text { toise) }) / \mathrm{kg} \text {. }\end{array}$} \\
\hline \multicolumn{3}{|c|}{$\begin{array}{l}\text { †Bacterial counts were expressed as mean (SD) } \log _{10} \text { counts/g wet weight, six rats per } \\
\text { group. }\end{array}$} \\
\hline \multicolumn{3}{|c|}{$\S$ Significantly different from standard diet $(P<0.05)$} \\
\hline
\end{tabular}



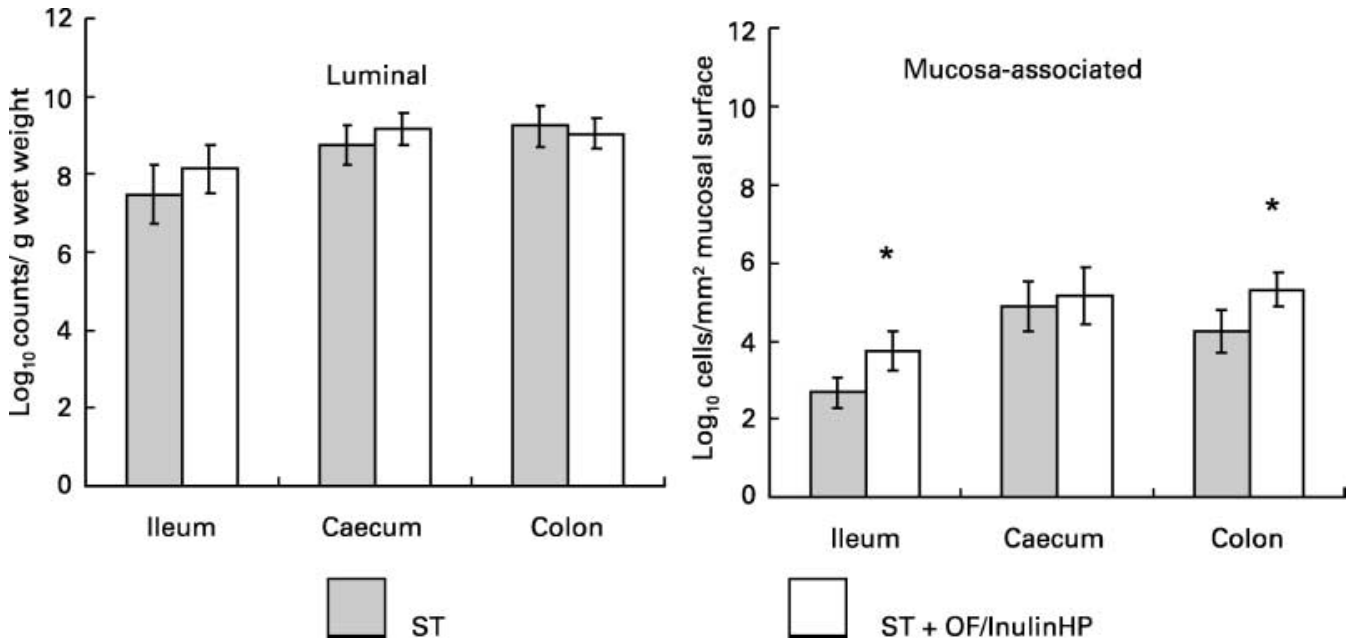

Fig. 6. Numbers of luminal $v$. mucosa-associated bifidobacteria in the ileum, caecum and colon of human flora-associated rats fed a standard diet (ST) or a standard diet supplemented with inulin-type fructans (OF, oligofructose; Inulin HP, high-molecular-weight inulin) $15 \mathrm{~d}$ after oral infection with a single dose of Salmonella enterica sub sp. enterica serovar Typhimurium. Mean values were significantly different from those of the ST group: ${ }^{\star} P<0.05$.

an important topic in the treatment and prophylaxis of gastrointestinal disorders and health maintenance.

\section{Inulin-type fructans affect the survival of Salmonella in human flora-associated rats}

A second study aimed to prove the efficacy of an inulin-type fructan-containing diet in reducing or preventing the colonization of Salmonella enterica subsp. enterica serovar Typhimurium (S. Typhimurium), a gut pathogen, in HFA rats. Therefore, HFA rats were randomly assigned to either control (ST) or ST supplemented with $5 \%$ of the mixture OF/inulin HP. Ten days later all rats were orally infected with a single dose of $S$. Typhimurium $\left(4 \times 10^{8}\right.$ viable cells). Colonisation of the ileum, caecum, and colon (intestinal content, mucus, mucosa surface) as well as organ invasion were evaluated for Salmonella on day 15 after infection by cultural methods and FISH. The possible fructan-induced stimulation of luminal and/or mucosa-associated bifidobacteria was also of particular interest.

The results indicated that high numbers of $S$. Typhimurium were detected in the mesenteric lymph nodes and the Peyer's patches of the infected rats (Table 1). A proportion of these organisms appeared to be bound to the ileal epithelium or mucus layer. The inulin-type fructans in the diet inhibited the survival of Salmonella in the intestinal contents but did not markedly reduce its mucosal translocation, although lower numbers of this organism were found in the Peyer's patches of rats consuming the $\mathrm{OF} /$ inulin HP-containing diet compared with ST. In this context it is noteworthy that the inulin-type fructan-treated group harboured more ileal and colonic mucosa-associated bifidobacteria than the non-treated ST group (Fig. 6), whereas the numbers observed in the intestinal contents were not statistically different. The finding of more bifidobacteria in the mucosal mucus layer of rats fed inulin-type fructans might be associated with a loss of adhesion sites for Salmonella. However, the observation that inulin-type fructans at a concentration of $5 \%$ in the diet were only partly effective against mucosal translocation of $S$. Typhimurium led us to speculate that the protective effects of these prebiotics could be dose-dependent. In addition, we assume that other factors such as the host immune responses, both humoral and cellmediated, are required to explain the protective effects of the inulin-type fructans against this enteric pathogen. It is tempting to speculate that the local immune response was involved in the reduction of $S$. Typhimurium in the Peyer's patches of the HFA rats fed inulin-type fructans.

\section{Conclusion}

Modulation of gut mucosal biofilms with inulin-type fructans to favour the growth and activity of potentially health-promoting bacteria such as bifidobacteria and thereby to alter the colonisation conditions of these organisms by means of changes in the mucosal architecture, release of mucins and/or stimulation of sulfomucins may be a way to protect the intestinal epithelium and to normalise a disturbed mucosal barrier. However, without knowing the exact mechanisms of biofilm formation and its maintenance, it is impossible to understand the relationship between the observed changes in the community structure and their function consequences.

The relevance in man of the above effects found in HFA rats remains to be shown.

\section{Acknowledgement}

This work was supported by European Research Project FAIRCT-97-3035.

\section{References}

Amann R \& Kühl M (1998) In situ methods for assessment of microorganisms and their activities. Curr Opin Microbiol 1, 352-358.

Amann RI, Krumholz L \& Stahl DA (1990) Fluorescent-oligonucleotide probing of whole cells for determinative phylogenetic, and environmental studies in microbiology. J Bacteriol 172, 762-770.

Amann RI, Ludwig W \& Schleifer K-H (1995) Phylogenetic identification and in situ detection of individual microbial cells without cultivation. Microbiol Rev 59, 143-169.

Berg RD (1996) The indigenous gastrointestinal microflora. Trends Microbiol 4, 430-435.

Bouhnik Y, Vahedi K, Achour L, et al. (1999) Short-chain fructo-oligosaccharide administration dose-dependently increases fecal bifidobacteria in healthy humans. J Nutr 129, 113-116. 
Campbell JM, Fahey GC \& Bryan WW (1997) Selected indigestible oligosaccharides affect large bowel mass, cecal and fecal short-chain fatty acids, pH and microflora in rats. J Nutr 127, 130-136.

Cummings JH \& Macfarlane GT (1997) Colonic microflora: nutrition and health. Nutrition 13, 476-478.

Deplancke B \& Gaskins HR (2001) Microbial modulation of innate defense: goblet cells and the intestinal mucus layer. Am J Clin Nutr 73, Suppl., 1131S-1141S.

Djouzi Z \& Andrieux C (1997) Compared effects of three oligosaccharides on metabolism of intestinal microflora in rats inoculated with a human faecal flora. Br J Nutr 78, 313-324.

Fontaine N, Meslin JC, Lory S \& Andrieux C (1996) Intestinal mucin distribution in the germ-free rat and in the heteroxenic rat habouring a human bacterial flora: effect of inulin in the diet. Br J Nutr 75, 881-892.

Forstner JF (1978) Intestinal mucins in health and disease. Digestion 17, 234-263.

Franks AH, Harmsen HJM, Raangs GC, Jansen GJ, Shut F \& Welling GW (1998) Variation of bacterial populations in human feces measured by fluorescent in situ hybridization with group-specific 16S rRNA-targeted oligonucleotide probes. Appl Environ Microbiol 64, 3336-3345.

Freitas M \& Cayuela C (2000) Microbial modulation of host intestinal glycosylation patterns. MEHD 12, Suppl. 2, 165-178.

Fuller R \& Gibson GR (1997) Modification of the intestinal microflora using probiotics and prebiotics. Scand J Gastroenterol 32, Suppl. 222, 28-31.

Gibson GR, Beatty EB, Wang X \& Cummings JH (1995) Selective stimulation of bifidobacteria in the human colon by oligofructose and inulin. Gastroenterology 108, 975-982.

Hooper LV \& Gordon JI (2001) Commensal host-bacterial relationships in the gut. Science 292, 1115-1118.

Kleessen B, Sykura B, Zunft H-J \& Blaut M (1997) Effects of inulin and lactose on fecal microflora, microbial activity, and bowel habit in elderly constipated persons. Am J Clin Nutr 65, 1397-1402.

Kleessen B, Hartmann L \& Blaut M (2001) Oligofructose and long-chain inulin: influence on the gut microbial ecology of rats associated with a human faecal flora. Br J Nutr 86, 291-300.

Kleessen B, Kroesen AJ, Buhr HJ \& Blaut M (2002) Mucosal and invading bacteria in patients with inflammatory bowel disease compared with controls. Scand J Gastroenterol 37, 1034-1041.

Kleessen B, Hartmann L \& Blaut M (2003) Fructans in the diet cause alterations of intestinal mucosal architecture, released mucins and mucosa-associated bifidobacteria in gnotobiotic rats. $\mathrm{Br} J$ Nutr 89, 597-606.

Kruse H-P, Kleessen B \& Blaut M (1999) Effects of inulin on faecal bifidobacteria in human subjects. Br J Nutr 82, 375-382.

Le Blay G, Michel C, Blottiere HM \& Cherbut C (1999) Prolonged intake of fructo-oligosaccharides induces a short-term elevation of lactic acid-producing bacteria and a persistent increase in cecal butyrate in rats. $J$ Nutr 129, 2231-2235.

Lu L \& Walker WA (2001) Pathologic and physiologic interactions of bacteria with the gastrointestinal epithelium. Am J Clin Nutr 73, Suppl., $1124 \mathrm{~S}-1130 \mathrm{~S}$.

Macfarlane S, McBain AJ \& Macfarlane GT (1997) Consequences of biofilms and sessile growth in the large intestine. Adv Dent Res 11, 59-68

Macfarlane S, Cummings JH \& Macfarlane GT (1999) Bacterial colonisation of surfaces in the large intestine. In Colonic Microbiota, Nutrition and Health, pp. 71-87 [GR Gibson and MB Roberfroid, editors]. Netherlands: Kluwer Academic Publisher.

Neish AS (2002) The gut microflora and intestinal epithelial cells: a continuing dialogue. Microbes Infect 4, 309-317.

Pluske JR, Tompson MJ, Atwood CS, Bird PH, Williams IH \& Hartmann PE (1996) Maintenance of villus height and crypt depth, and enhancement of disaccharide digestion and monosaccharide absorption, in piglets fed on cows' whole milk after weaning. $\mathrm{Br} J \mathrm{Nutr} \mathbf{7 6}$, 409-422.

Pullan RD, Thomas GA, Rhodes M, Newcombe RG, Williams GT, Allen A \& Rhodes J (1994) Thickness of adherent mucus gel on colonic mucosa in humans and its relevance to colitis. Gut 35, 353-359.

Rhodes JM (1997) Mucins and inflammatory bowel disease. Q J Med 90, 79-82.

Romeis R (1989) Polysaccharide and mucous substances. In Microscopic Technique, pp. 439-444 [P Böck, editor]. Munich, Vienna, Baltimore: Urban \& Schwarzenberg.

Sakata T (1987) Stimulatory effect of short-chain fatty acids on epithelial cell proliferation in the rat intestine: a possible explanation for trophic effects on fermentable fibre, gut microbes and luminal trophic factors. Br J Nutr 58, 95-103.

Salminen S, Isolauri E \& Onnela T (1995) Gut flora in normal and disordered states. Chemotherapy 41, Suppl. I, 5-11.

Tolker-Nielsen T \& Molin S (2000) Spatial organization of microbial biofilms communities. Microb Ecol 40, 75-84. 